\title{
Whole genome sequencing of one complex pedigree illustrates challenges with genomic medicine
}

Han Fang ${ }^{1,2,3+}$, Yiyang Wu ${ }^{1,2+}$, Hui Yang ${ }^{7,8}$, Margaret Yoon ${ }^{1}$, Laura T. Jiménez-Barrón ${ }^{1,4}$, David Mittelman5, Reid Robison ${ }^{5,6}$, Kai Wang ${ }^{7,9,10,11}$ and Gholson J. Lyon ${ }^{1,2,6^{*}}$

\begin{abstract}
Background: Human Phenotype Ontology (HPO) has risen as a useful tool for precision medicine by providing a standardized vocabulary of phenotypic abnormalities to describe presentations of human pathologies; however, there have been relatively few reports combining whole genome sequencing (WGS) and HPO, especially in the context of structural variants.

Methods: We illustrate an integrative analysis of WGS and HPO using an extended pedigree, which involves Prader-Willi Syndrome (PWS), hereditary hemochromatosis $(\mathrm{HH})$, and dysautonomia-like symptoms. A comprehensive WGS pipeline was used to ensure reliable detection of genomic variants. Beyond variant filtering, we pursued phenotypic prioritization of candidate genes using Phenolyzer.

Results: Regarding PWS, WGS confirmed a 5.5 Mb de novo deletion of the parental allele at 15q11.2 to 15q13.1. Phenolyzer successfully returned the diagnosis of PWS, and pinpointed clinically relevant genes in the deletion. Further, Phenolyzer revealed how each of the genes is linked with the phenotypes represented by HPO terms. For HH, WGS identified a known disease variant (p.C282Y) in HFE of an affected female. Analysis of HPO terms alone fails to provide a correct diagnosis, but Phenolyzer successfully revealed the phenotype-genotype relationship using a disease-centric approach. Finally, Phenolyzer also revealed the complexity behind dysautonomia-like symptoms, and seven variants that might be associated with the phenotypes were identified by manual filtering based on a dominant inheritance model.

Conclusions: The integration of WGS and HPO can inform comprehensive molecular diagnosis for patients, eliminate false positives and reveal novel insights into undiagnosed diseases. Due to extreme heterogeneity and insufficient knowledge of human diseases, it is also important that phenotypic and genomic data are standardized and shared simultaneously.
\end{abstract}

Keywords: Whole genome sequencing, Precision medicine, Human phenotype ontology, Phenolyzer, Variant calling, Prader-Willi Syndrome, Dysautonomia, Hemochromatosis

\footnotetext{
* Correspondence: gholsonjlyon@gmail.com

${ }^{\dagger}$ Equal contributors

${ }^{1}$ Stanley Institute for Cognitive Genomics, One Bungtown Road, Cold Spring

Harbor Laboratory, Cold Spring Harbor, NY, USA

${ }^{2}$ Stony Brook University, 100 Nicolls Rd, Stony Brook, NY, USA

Full list of author information is available at the end of the article
} 


\section{Background}

Many genetic tests have been commonly performed on individuals that have phenotypes overlapping with known diseases, especially for cancer and rare diseases [1-4]. Physicians have also been routinely prescribing prenatal genetic tests and newborn screenings in clinics [5-7]. However, there is a degree of uncertainty inherent in most genetic testings regarding the development, age of onset, and severity of disease [8]. In addition, current genetic testing has not yet established predictive or even diagnostic value for common complex diseases [9]. Some groups have begun to leverage the power of nextgeneration sequencing (NGS) to help diagnose rare diseases [10-13]. Many studies have used whole exome sequencing (WES) to facilitate the molecular diagnosis of individuals with diseases that appear to have a single large-effect size mutation contributing substantially to the development of the disease, referred to by many as "Mendelian disorders" $[14,15]$. Of course, such disorders also have an extraordinary phenotypic variability and spectrum brought about by genetic background, environmental differences and stochastic developmental variation (SDV) [16-20].

Despite much success using NGS-based techniques to identify mutations, there are still practical issues for the analytic validity for exome- or genome-wide NGS-based techiques, particularly in clinical settings [21, 22]. The clinical utility of genomic medicine is also uncertain, prompting some to suggest the need for better standards and benchmarking $[23,24]$. However, the genetic architecture behind human disease is heterogeneous, and there are many reports of regulatory variants in the noncoding genome and splicing variants in the intronic regions that have a large-effect size on particular phenotypes [25-30]. In hypothesis-driven research studies, one might gain higher statistical power with a larger sample size by using cheaper NGS assays like WES or gene panels. But whole genome sequencing (WGS) has a unique strength in its ability to cover a broader spectrum of variants; small insertions and deletions (INDELs), structual variants (SVs), and copy number variants (CNVs). This becomes extremely valuable in studies where disease associated variants might not be necessarily SNVs [31-33]. In particular, from a study design perspective, WGS results in a more uniform coverage and better detection of INDELs, and is free of exome capture deficiency issues [34]. Of course, cost and technical considerations are still practical issues for WGS, but this will eventually become the optimal assay to address the extreme heterogeneity of different genetic architectures for different diseases.

Human Phenotype Ontology (HPO) has risen as a useful techique for precision medicine by providing a standardized vocabulary bank of phenotypic abnormalities to describe presentations of human pathologies [35-37]. Some showed that phenotypic matching can help interpret $\mathrm{CNV}$ findings based on integrated cross-species phenotypic information [38]. The potential clinical usage of HPO derives from a wealth of medical literature and databases such as Online Mendelian Inheritance in Man (OMIM) [35]. Computational tools like Exomizer and PhenIX were developed to aid disease associated variant prioritization from exome sequencing data [39-41], and this has been recently extended with the development of Genomiser for WGS data [42]. Another tool is Phenolyzer [43], which uses prior biological knowledge and phenotype information to implicate genes involved in diseases. Phenolyzer reveals the hidden connection of genotypes and phenotypes by examing gene-gene, gene-disease and disease-phenotype interactions [43]. Based on standarized phenotypic reports, Phenolyzer can be used to further prioritize WGS findings for disease associated variants.

We report here a comprehensive analysis of an extended pedigree, including genomics filtering on WGS data and phenotypic prioritization of candidate genes using Phenolyzer. The pedigree involves probands with Prader-Willi Syndrome (PWS) [44, 45], Hereditary Hemochromatosis $(\mathrm{HH})$, dysautonomia-like symptoms, Tourette Syndrome (TS) [46] and other illnesses. We specifically chose this family for whole genome sequencing due to the phenotypic complexity in the family, including at least one genetic syndrome with a known genetic etiology, which on some level serves as a positive control among a range of diseases of unknown (or controversial) genetic architecture. Nine members of the family underwent WGS, enabling a wide scope of variant calling from SNVs to large copy number events. Notably, this is the first report of Illumina HiSeq WGS experiement on a PWS individual carrying the paternallyinherited deletion. The use of WGS enables the reconstruction of the recombination event in this imprinting hotspot, which provides a better understanding of the PWS disease mechanism. This report emphasizes the effectiveness of Phenolyzer, which can be used to integrate and share WGS and HPO data. Neither technique is yet perfect for clinical diagnosis, but combining the two can help eliminate false positives and reveal novel insights into human diseases.

\section{Methods}

Clinical phenotyping of individuals participating in this study

The family was interviewed by the corresponding author, GJL, a board-certified child, adolescent and adult psychiatrist. Medical records were obtained and reviewed, in conjunction with further interviews with the family. The interviews were videotaped and later reviewed to 
facilitate further diagnostic efforts. Various clinical diagnostic testings were performed on K10031-10133, including tilt table test, brain MRI, ultrasound of the kidneys and chest X-ray. In addition, her cholesterol level, thyroid profile, urine vanillylmandelic acid (VMA), catecholamines panel (urine-free), basic metabolic panel (BMP), and epinephrine and norepinephrine levels were also screened. Other clinical tests included electrocardiogram (EKG), polysomnographic report, and echocardiogram. For K10031-10232, the following diagnostic evaluations were performed: multiple sleep latency test (MSLT) [47], autism diagnostic observation system (ADOS) - module 2 [48], the Childhood Autism Rating Scale (CARS) [49], Behavior Assessment System for Children (BASC) [50], Intelligence Quotient (IQ), and Abnormal Involuntary Movement Scale (AIMS) [51].

\section{Generation of WGS and microarray data}

Blood and saliva samples were collected from nine individuals (K10031-10143, 10144, 10145, 10235, 10133, $10138,10231,10232,10233)$ from the extended pedigree described in the results. Two CLIA-certified WGS tests (K10031-10133 and K10031-10138) were performed at Illumina, San Diego. The other seven WGS runs were performed at the sequencing center at Cold Spring Harbor Laboratory (CSHL). All libraries were constructed with PCR amplification, and sequenced on one Illumina HiSeq2000 with an average paired-end read length of $100 \mathrm{bp}$. Since the DNA extracted from saliva samples contains a certain proportion of bacterial DNA, these samples were sequenced on additional lanes to achieve an average coverage of $40 \mathrm{X}$ after removing unmapped reads (Additional file 1: Table S1). Microarray data for the same samples were generated with the Illumina Omni 2.5 microarray at the Center for Applied Genomics Core of the Children's Hospital of Philidephia (CHOP). Illumina Genome Studio was used to extract the SNV calls and $\log \mathrm{R}$ ratio (LRR) and $\mathrm{B}$ allele frequency (BAF) from the microarray data. The general analysis work-flow is shown in Additional file 1: Fig. S1.

\section{Alignment and variant calling of WGS data}

All of the unmapped raw reads were excluded to remove the sequence reads coming from the bacterial DNA (step 2 of Additional file 1: Fig. S1). The remaining reads were aligned to human reference genome (build hg19) with BWA-mem (v0.7-6a) [52]. In parallel, reads were also aligned with NovoAlign (v3.00.04) to reduce false negatives resulting from alignment artifacts. All of the alignments were sorted with SAMtools (v0.1.18) and PCR duplicates marked with Picard (v1.91) [53]. For the BWA-MEM bam files, INDELs were realigned with the GATK IndelRealigner (v2.6-4) and base quality scores were recalibrated [54]. For variant calling with FreeBayes, the alignment files were not processed with INDELrealignment and base quality recalibration as these additional steps are not required by FreeBayes. Qualimap (v2.0) was used to perform QC analysis on the alignment files [55].

In order not to miss potentially disease-contributory variants, more than one pipeline were used to detect SNVs, INDELs, SVs, and CNVs [56, 57]. All variants are included in the downstream analysis and orthogonal validations were performed to confirm the variants of interest (step 3 to step 5 of Additional file 1: Fig. S1). First, SNVs and INDELs were jointly called from nine genomes with GATK HapolotypeCaller (v3.1-1) from the BWA-MEM alignment following best practices [58]. Second, a default parameter setting was used to call variants using FreeBayes from the NovoAlign alignment [59]. Third, Scalpel (v0.1.1) was used with the BWAMEM bam files to identify INDELs in the exonic regions with sizes up to $100 \mathrm{bp}$ [60]. Each exon was expanded by $20 \mathrm{bp}$ upstream and $20 \mathrm{bp}$ downstream to reveal possible INDELs harboring splicing sites. Following the benchmarking results as recently reported [34], Scalpel INDEL calls were filtered out if they have an alternative allele coverage less than five and a Chi-Square socre greater than 10.8. Fourth, RepeatSeq (v0.8.2) was utilized to detect variants near short tandem repeats regions in the genome using default settings [61]. Fifth, Lumpy (v 0.2.6) and CNVnator were both used to call SVs with sizes $>100$ bp $[62,63]$. Among Lumpy calls, events supported by $>50$ reads or less than four reads were excluded because regions of either too low or high coverage are more likely to contain biases in sequencing or alignment. Sixth, ERDS (v1.1) was used to call CNVs from the BWA-mem bam files with default settings [64]. Among ERDS calls with a confidence score $>300$, duplications with sizes $<200 \mathrm{~Kb}$ and deletion calls with sizes $<10 \mathrm{~Kb}$ were excluded from downstream analysis. CNVnator (v0.3) was used to identify smaller CNVs that are present in the WGS data using the parameters -his 100,-stat 100, -partition 100 , -call 100 [63]. Sixth, to achieve high confidence CNV calls, PennCNV (2011Jun16 version) was used to call CNVs from the microarray data [65]. Each CNV was supported by at least 10 markers, excluding CNVs with an inter-marker distance of $>50 \mathrm{~Kb}$. SVs and CNVs that overlapped with segmental duplication regions by $50 \%$ were also filtered out with BEDtools [66].

\section{Genomic filtering and annotations of the variants}

To annotate the variants of interest, GEMINI (v0.11.0), ANNOVAR (2013Aug23 version) were used (step 6 of Additional file 1: Fig. S1) [67, 68]. The circos plot of K10031-10232's genome was generated using circlize in 
$\mathrm{R}$ [69]. The population allele frequencies (AF) were loaded with GEMINI from the 1000G database (http:// www.1000genomes.org/) and Exome Aggregation Consortium (ExAC) database (http://exac.broadinstitute.org/) [70]. GEMINI also served to import the CADD C-scores, loss-of-function variants defined by LOFTEE, and the reported pathogenicity information from the ClinVar database [71, 72]. There were several steps in filtering variants with respect to the segregation pattern, population frequency, allele deleteriousness prediction, and ClinVar annotation. First, variants were partitioned by the following disease inheritance models: autosomal dominant, autosomal recessive, de novo, compound heterzygous, and X-linked dominant. Second, autosomal or X-linked dominant and de novo variants were excluded if they had an AAF $>0.01$ in either ExAC or $1000 \mathrm{G}$ database while the cut-off was increased to 0.05 for autosomal recessive and compound heterzygous variants. Third, only the variants that met the following criteria were considered in the downstream analysis: 1) called by at least one pipeline and validated with a second pipeline, 2) had an adjusted p-value lower than 0.05 reported by pVAAST [73], 3) defined as medium or high impact by GEMINI, or defined as loss-of-function by LOFTEE, 4) with a CADD c-score greater than 15. Fourth, we also searched for variants that were considered as pathogenic, probably-pathogenic, mixed, or drug-response in the ClinVar database. Lastly, the VCF files were also uploaded to the Omicia Opal platform and the Tute Genomics platform for online annotation, filtering, and pharmacogenomic analysis. The Tute Genomics variant interpretation report for each individual can be found in Additional file 2.

\section{Phenotypic prioritization of candidate genes using Phenolyzer}

Clinical features of K10031-10232, K10031-10133, and K10031-10145 were mapped to HPO terms using the Phenomizer clinical diagnostics tool [74]. Complete Phenomizer diagnosis forms are available in supplemental files. Phenolyzer was used for phenotypic prioritization of the genomic variants in above three probands. For each proband, we first performed a genomic filtering of the WGS data, compiling a list of candidate genes and genomic intervals of SVs and CNVs. Then we uploaded the filtered list and their HPO terms to Phenolyzer for phenotypic prioritization. HPO terms were generated for K10031-10232, K1003110145, K10031-10133 (see Table 1, Table 2, Additional file 1: Supplemental Table S5, S6, and S10).

To find out what HPO terms affect our results the most, we performed a ranking analysis with Phenolyzer. We used individual HPO term as input and compared the Phenolyzer scores of the CNV containing NDN
Table 1 Main Clinical Presentation of Proband K10031-10232

\begin{tabular}{|c|c|}
\hline Clinical manifestations & HPO\# \\
\hline \multicolumn{2}{|l|}{ Development and growth } \\
\hline Delayed speech and language development & 0000750 \\
\hline Growth hormone deficiency & 0000824 \\
\hline Poor fine motor coordination & 0007010 \\
\hline Mild intellectual disability & 0001256 \\
\hline \multicolumn{2}{|l|}{ Facial features } \\
\hline Almond-shaped eyes & 0007874 \\
\hline Downslanted palpebral fissures & 0000494 \\
\hline Narrow forehead & 0000341 \\
\hline \multicolumn{2}{|l|}{ Other physical features } \\
\hline Cryptorchidism & 0000028 \\
\hline Excessive daytime sleepiness & 0002189 \\
\hline Obstructive sleep apnea syndrome & 0002870 \\
\hline Scoliosis & 0002650 \\
\hline \multicolumn{2}{|l|}{ Behavior features } \\
\hline Aggressive behavior & 0000718 \\
\hline Anxiety & 0000739 \\
\hline Depression & 0000716 \\
\hline Impaired ability to form peer relationships & 0000728 \\
\hline Impaired social reciprocity & 0012760 \\
\hline Inflexible adherence to routines or rituals & 0000732 \\
\hline Irritability & 0000737 \\
\hline Low frustration tolerance & 0000744 \\
\hline Obsessive-compulsive disorder & 0000722 \\
\hline Pain insensitivity & 0007021 \\
\hline Polyphagia & 0002591 \\
\hline Poor eye contact & 0000817 \\
\hline Restrictive behavior & 0000723 \\
\hline Short attention span & 0000736 \\
\hline
\end{tabular}

and SNRPN. Ideally, the higher the score, the more important this HPO term is to this CNV. Further, to understand the impact of the number of HPO terms on the final result, we randomly downsampled to a smaller number (one to six) of HPO terms from the entire set of 21. Then we used each combination as an input for Phenolyzer analysis. We defined the confidence level of a result based on the Phenolyzer score of the correct CNV; 'High confidence' ( $>=0.5)$, 'Medium confidence' $(0.1=<$ Phenolyzer score $<0.5)$ and 'Low confidence' $(<0.1)$. For each scenario (one to six HPO terms), we counted the number of times when the correct $\mathrm{CNV}$ was prioritized at high/medium/ low confidence levels. Finally, we computed and summarized the percentage of each (Fig. 6). 
Table 2 Main Clinical Presentation of Proband K10031-10133

\begin{tabular}{|c|c|}
\hline Clinical manifestations & HPO\# \\
\hline \multicolumn{2}{|l|}{ Cardiovascular } \\
\hline Bradycardia & 0001662 \\
\hline Patent foramen ovale & 0001655 \\
\hline Syncope & 0001279 \\
\hline Tachycardia & 0001649 \\
\hline \multicolumn{2}{|l|}{ Eyes } \\
\hline Diplopia & 0000651 \\
\hline Peripheral vision & NF \\
\hline \multicolumn{2}{|l|}{ Gastrointestinal } \\
\hline Gastroparesis & 0002578 \\
\hline Nausea & 0002018 \\
\hline \multicolumn{2}{|l|}{ Gynecologic \& genitourinary } \\
\hline Urinary retention & 0000016 \\
\hline Urinary incontinence & 0000020 \\
\hline \multicolumn{2}{|l|}{ Musculoskeletal } \\
\hline Arthralgia & 0002829 \\
\hline Joint stiffness & 0001387 \\
\hline \multicolumn{2}{|l|}{ Neurological } \\
\hline Apraxia & 0002186 \\
\hline Arthritis & 0001369 \\
\hline Auditory hallucinations & 0008765 \\
\hline Convulsions & $\mathrm{NF}$ \\
\hline Dizziness & NF \\
\hline Dysarthria & 0001260 \\
\hline Fatigue & 0012378 \\
\hline Frequent falls & 0002359 \\
\hline Heat intolerance & 0002046 \\
\hline Migraine & 0002076 \\
\hline Seizure & 0001250 \\
\hline Tremor (Postural/Resting) & $0002174 / 0002322$ \\
\hline Visual hallucinations & 0002367 \\
\hline \multicolumn{2}{|l|}{ Respiratory } \\
\hline Asthma & 0002099 \\
\hline \multicolumn{2}{|l|}{ Psychiatric } \\
\hline Anxiety & 0000739 \\
\hline Depression & 0000716 \\
\hline
\end{tabular}

Abbreviation: $N F$, Not found. For a full version of the table, please refer to Additional file 1: Table $\mathrm{S} 6$

\section{Results and discussion}

Clinical presentation (with HPO annotation) and family history

Here, we present the phenotypic characterization of a Utah pedigree K10031, consisting of 14 individuals from three generations (Fig. 1) with various medical conditions as mentioned above. The two probands we discuss in detail below come from two nuclear families in this extended pedigree.

\section{Proband K10031-10232}

Proband K10031-10232 is a 25-year-old (25 y.o.) male. $\mathrm{He}$ is the son of a Caucasian farther (K10031-10231), and an Asian mother (did not participate in the study). He has two older male siblings, namely K10031-10233 and K10031-10234. This proband was diagnosed with PWS at 11 months old, and has dysmorphic facial features including a narrow forehead, downslanted palpebral fissures and almond-shaped eyes. A description of a video recording (HDV_0073) illustrating his clinical manifestations can be found in the supplemental section, and the video can be provided on request to qualified investigators. Since the PWS diagnosis, his behavior has been assessed in great detail (Table 1, and Additional file 1: Supplemental Data), and the following diagnoses have been given: obsessive-compulsive disorder (OCD), depression, anxiety disorder, pervasive developmental disorder (PDD), hyperphagia, trichotillomania, and daytime hypersomnolence. He has an IQ ranging between 60 and 65, categorized as mild mental retardation. He also has diagnoses of mild dysarthria, obstructive sleep apnea syndrome (OSAS), and severe scoliosis. The latter has been corrected surgically. He has also undergone orchiopexy, tonsillectomy, and adenoidectomy. His physical exam is otherwise unremarkable. He has denied having significant psychotic symptoms, including auditory or visual hallucinations, delusions, ideas of grandiosity, or paranoid ideation.

In an effort to help standardize phenotype reporting, we used Human Phenotype Ontology (HPO) annotation [75]. See Table 1 and Additional file 1: Table S5 for a list of clinical phenotype features collected from this proband. The Phenomizer tool [74] ranked the diagnosis for Prader-Willi Syndrome as the highest priority diagnosis for this proband (see Additional file 3), supporting the fact that highly specific and annotated phenotype information can yield accurate diagnoses, at least for a characteristic syndrome like PWS. As presented below, the genomic analysis of proband K10031-10232 further confirmed deletions in the chromosome regions from $15 q 11.2$ to $15 q 13.1$, making PWS the most credible diagnoses for him at present.

\section{Proband K10031-10133}

Proband K10031-10133 is a 26 y.o. female, born to a Caucasian mother (K10031-10145) and a Caucasian father (K10031-10144). She is the eldest child amongst her two sisters and two brothers. Prior to age 18, K10031-10133 had a fairly unremarkable medical history. Arthralgia and episodes of fatigue and dizziness 


\section{Pedigree K10031}

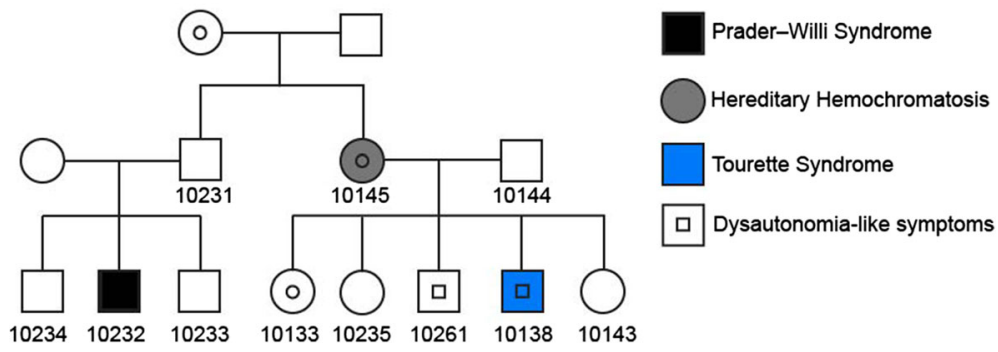

Fig. 1 A pedigree spanning three generations with multiple diseases in this study. DNA was collected with informed consent from individuals marked with a number underneath. All samples underwent WGS except for K10031-10234 and K10031-10261

started at around 18 years of age. At age 20, she started to have refractory syncopal events, which led to multiple body injuries. During the same period of time, she also developed postural orthostatic tachycardia syndrome (POTS), heart palpitations, gastroparesis, urinary incontinence, diplopia, and seizures. In addition, she reported experiencing auditory and visual hallucinations. She underwent dysautonomia evaluation and revealed a positive tilt table test. Other tests revealed unusual changes to her optic disks but without an elevated intraocular pressure, and nonspecific findings on her brain MRI, including a subtle focus of T2 signal abnormality involving the subcortical white matter of the right parietal lobe without associated enhancement. See Table 2 and Additional file 1: Table S6 for proband K10031-10133's clinical phenotype list with HPO annotations, and Additional file 1: Supplemental Data for a full report of HPO analysis on her. Descriptions of video recordings (HDV_0079) of this proband illustrating her medical presentation and (HDV_0072) in which conditions in other family members are discussed are included in the supplemental videos section, and these videos can be provided on request to qualified investigators.

As for her family history (Additional file 1: Table S6), there are some noticeable symptoms that are shared by all her siblings and her mother, including dysautonomialike symptoms such as dizziness and fainting, as well as tremors and asthma. In addition, anxiety, attention deficit, arthritis, dyslexia, gastroesophageal reflux, seizures and TS are other diagnoses found among her siblings. Her mother (K10031-10145), on the other hand, has HH and OCD traits. Her father has significant migraines, gastroesophageal reflux, hiatal hernia, and right sensorineural hearing loss. See detailed descriptions of her family members in Additional file 1: Supplemental Data. We are highlighting here that extensive characterization of families, including videotaping and the collection of collateral information from other relatives, yields a rich texture of findings that are not always easily captured in written medical records.

\section{Summary statistics of the WGS data}

We previously reported a large false negative rate with the Complete Genomics platform [56], so we chose to utilize the Illumina platform for WGS. Nine members of the family underwent WGS, enabling a wide scope of variant calling from a single SNV to large CNVs. To reduce false variant calls, more than one pipeline were used to detect SNVs, INDELs, large SVs, and CNVs, as we previously suggested [56] (Fig. 2). Summary statistics for the WGS data for each sample are reported in Additional file 1: Table S1, S2, and Fig. S2. The average number of reads per sample is $1,432,506,869$. The number of mapped bases per sample is $124,410,724,287$, with a mean coverage of the WGS data across the genome of about 40X ( $89 \%$ of the bases in the genome covered with at least 20X). The insert size of the libraries is about 338 and the GC content is approximately $40 \%$ across samples. With the WGS data, a mean number of 4,099,604 $(\mathrm{SD}=47,076) \quad$ SNVs, $896,253 \quad(\mathrm{SD}=14327) \quad$ INDELs, $1,284(\mathrm{SD}=103) \mathrm{SVs}$, and $61(\mathrm{SD}=4) \mathrm{CNVs}$ are detected across nine samples (Additional file 1: Table S2). Within the coding regions, the average number of SNVs, INDELs, SVs, and CNVs detected are 22,406, 2,812, 511, 12, respectively. Kinship between individuals was inferred with KING to confirm family relationship between research participants in this study (Additional file 1: Table S3) [76].

\section{WGS identified de novo CNV deletions in $15 q 11.2$ to $15 q 13.1$ of proband K10031-10232}

ERDS and CNVnator both detected three de novo heterozygous deletions with a total size of about $5.5 \mathrm{Mb}$, in the chromosome regions from $15 \mathrm{q} 11.2$ to 15q13.1 of the proband with PWS (K10031-10232) (Fig. 3). The hg19 genomic coordinates of the breakpoints are chr15:22,749,401-23,198,800 ( 449 Kb), chr15:23,608,601-28,566,000 ( 4.96 Mb), and chr15: 28,897,601-28,992,600 ( 95 Kb). Notably, these deletions are relatively close to one another; the distances between each deletion are $\sim 410 \mathrm{~Kb}$ and $\sim 332 \mathrm{~Kb}$, respectively. 
R A

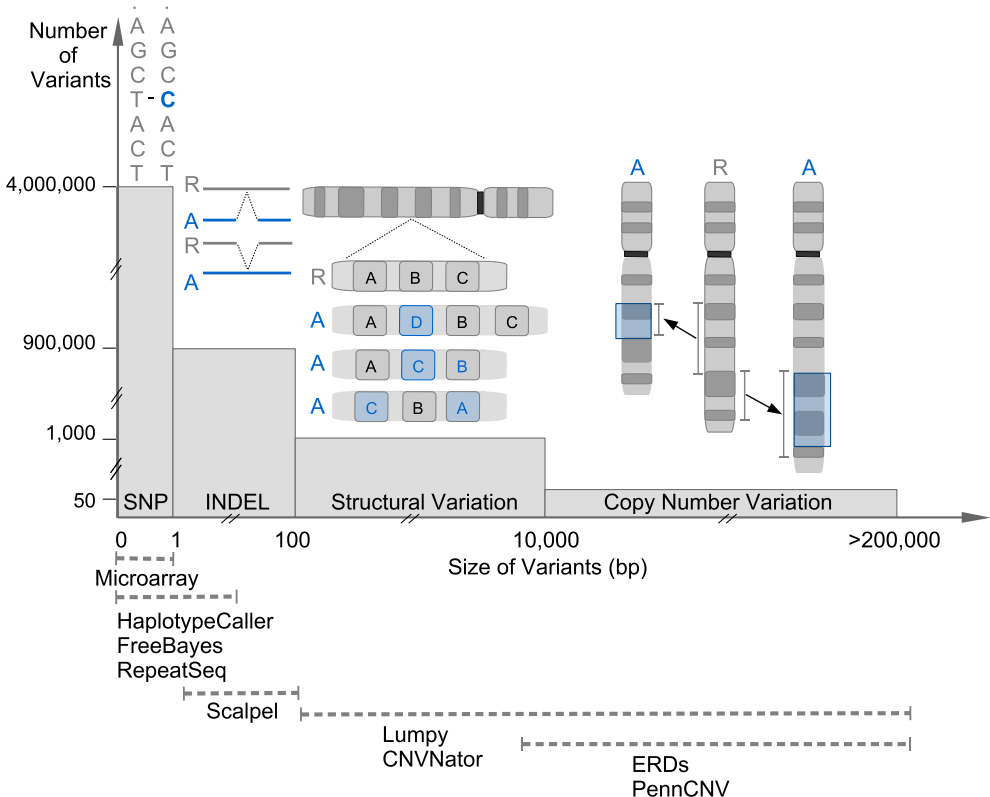

Fig. 2 WGS can reveal a broad spectrum of variants with softwares that are specialized for different types of variants. This is a conceptual illustration of variations in the human genome. The $Y$-axis shows the approximate number of variants in that category while the $X$-axis shows the approximate size of those variants. The interval below shows that variants of different sizes and sequence compositions can be better detected by leveraging the strength of different callers. SNV: single-nucleotide variation, INDEL: insertions and deletion, SV: structural variant, CNV: copy number variant

Within the regions containing the de novo deletions, the depth of coverage in the proband's genome is 20X, about half of the genome-wide mean coverage (40X). Due to the lack of the proband's mother's sequencing data (as she refused to participate), analysis was performed to determine which allele (paternal or maternal) is deleted. This can be inferred through SNVs where the mendelian inheritance law is violated; meaning those instances in which the proband (K10031-10232) does not carry certain paternal or maternal SNVs that his brother (K10031-10233) does carry. In total, there are 2,987 SNVs where the proband's father (K10031-10231) is a homozygote and the proband's brother is a heterozygote. Out of the 2112 SNVs where the father is homozygous to the reference allele, the proband is homozygous to the alternative allele at 1944 loci (92\%, Fig. 4). Among 875 SNVs where the father does not carry any reference allele, the proband carries only the reference allele at 861 SNVs (94\%, Fig. 4). This indicates that the proband only carries the maternal alleles in those regions. These deletions were not detected in either the proband's father or his brother using the WGS data (Additional file 1: Fig. S3). The Illumina microarray data further confirmed this discovery; the proband carries these deletions (Additional file 1: Fig. S4) while his father and his brothers (K10031-10233 and K10031-10234) do not carry any of these deletions in their genome (Additional file 1: Fig. S4-S6). Probe distributions of Log-R ratios and $B$ allele frequencies are not uniform in the microarray because the density of SNV varies between genomic regions (Additional file 1: Fig. S4-S7). This highlights the higher resolution and completeness of WGS over microarray for precise molecular diagnosis of such diseases. Thus, we confirm that the proband carries the de novo PWS Type I deletion (spanning breakpoints BP1 and BP3) defined by previous publications $[77,78]$. The complete list of genes that fall into the deletion regions are described in Additional file 1: Table S7.

\section{Phenolyzer discovered interaction between PWS deletions and disease subtypes}

Phenolyzer accurately revealed the diagnosis of PWS and how genes in the deletion regions are linked towards the phenotypes represented by HPO terms. The Phenolyzer network analysis of gene findings, HPO terms, and diseases types are shown in Fig. 5. The most disease relevant genes are showed as seed genes. Among all genes in the deletion regions, SNRPN, NDN and thirteen other genes are the most confident genes, which are maternally imprinted and commonly deleted in PWS $[79,80]$. Further, yellow lines indicate that the two node genes are within the same biosystem while green lines indicate that the two genes are within the same gene 


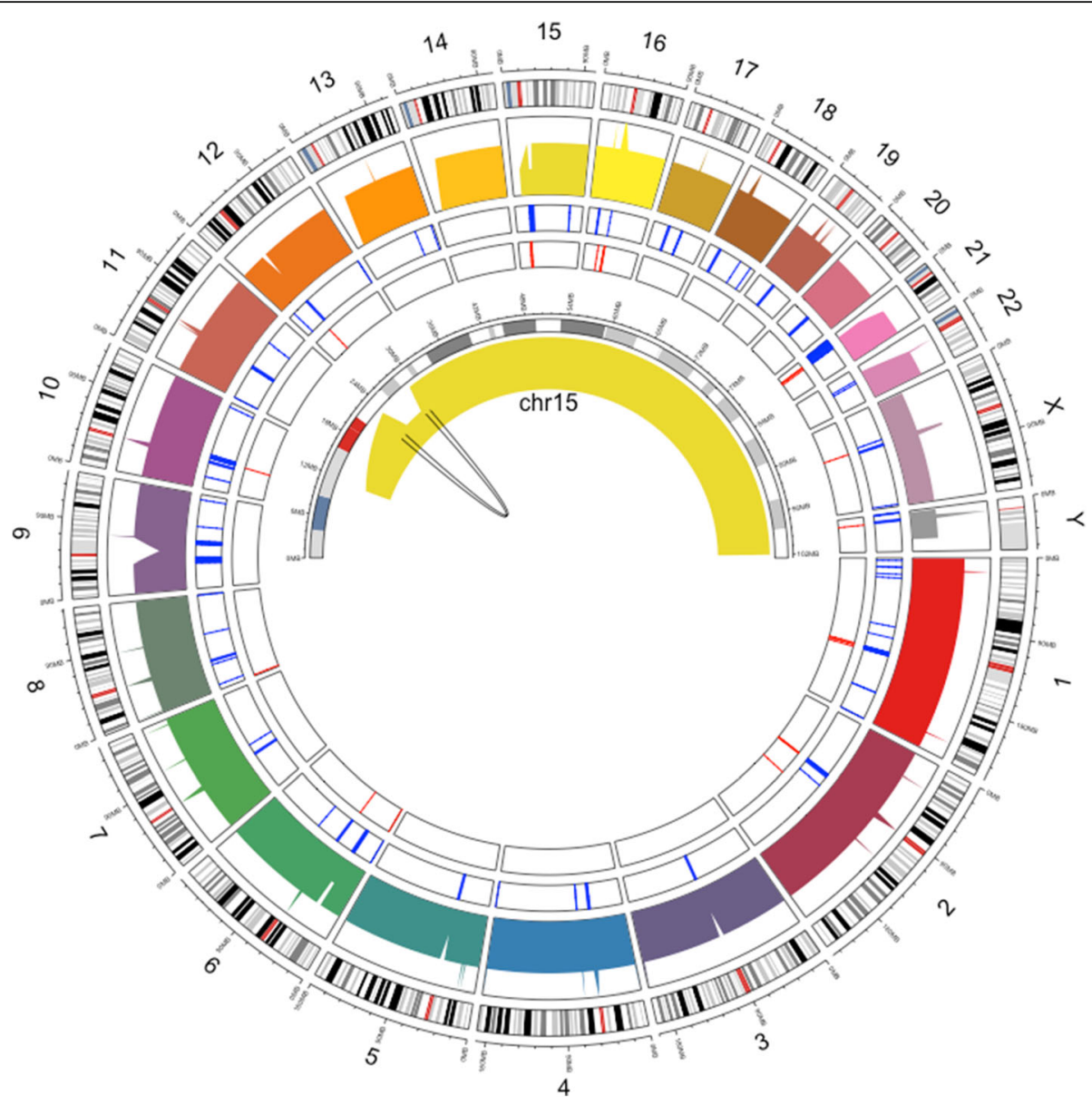

Fig. 3 Circos plot of the PWS proband's genome, highlighting chromosome 15. The outer circle is the cytoband of the human genome. The inner circle is the genome coverage of the PWS proband's (K10031-10232) genome. The breakpoint of the 15q11.2-15q13 deletion region in chromosome 15 is illustrated in the center

family. Phenolyzer reports two clusters of genes that are in the same Biosystem. The first cluster includes SNRPN, OR52N5, SNUPF, UBE3A, HERC2. The second cluster consists of GABRG3, GABRB3, GABRB5, ATP10A, while the former three genes are also from the same gene family.

Phenolyzer mapped nine HPO terms to PWS, including HP:0002591 (polyphagia), HP:0000824 (growth hormone deficiency), and HP:0007874 (almond shaped eyes). It is shown that the combination of the above three terms largely determine the diagnosis of PWS (Fig. 6). Phenolyzer score of the correct deletion corresponding to each HPO term is normalized to a range from 0 to 1 . A higher score indicates that this HPO term has a higher impact on the diagnosis of the corresponding disease. Both 'polyphagia' and 'growth hormone deficiency' have a Phenolyzer score of 1.0 and 'almond shaped eyes' has a score of 0.8 . Phenolyzer also reveals the molecular and phenotypic similarities between PWS and its related diseases. For example, Phenolyzer reports that Angelman Symdrome (AS) shares three HPO terms and eleven genes. Among those genes, $U B E 3 A$ has been implicated in AS due to a loss of gene expression from maternal chromosome [81]. Three overlapping phenotypic features are also discovered, including HP:0002650 (Scoliosis), HP:0000750 (Delayed speech and language development), and HP:0001999 (Abnormal facial shape).

Another interesting question is whether the number of input HPO terms used will impact the final result. To answer this, we conducted a series of combination analyses with one to six HPO terms out of the 21 candidate terms as input into Phenolyzer, respectively. We noticed that the more candidate terms we used, the more combinations we have. For example, the number of combinations for six out of 21 is 54,264 . Thus, due to the large overhead of computation time, we did not go beyond six terms. From the result, the more HPO terms we used as input, the higher the chance the known $\mathrm{CNV}$ was prioritized as 'High confidence' (Fig. 7). 


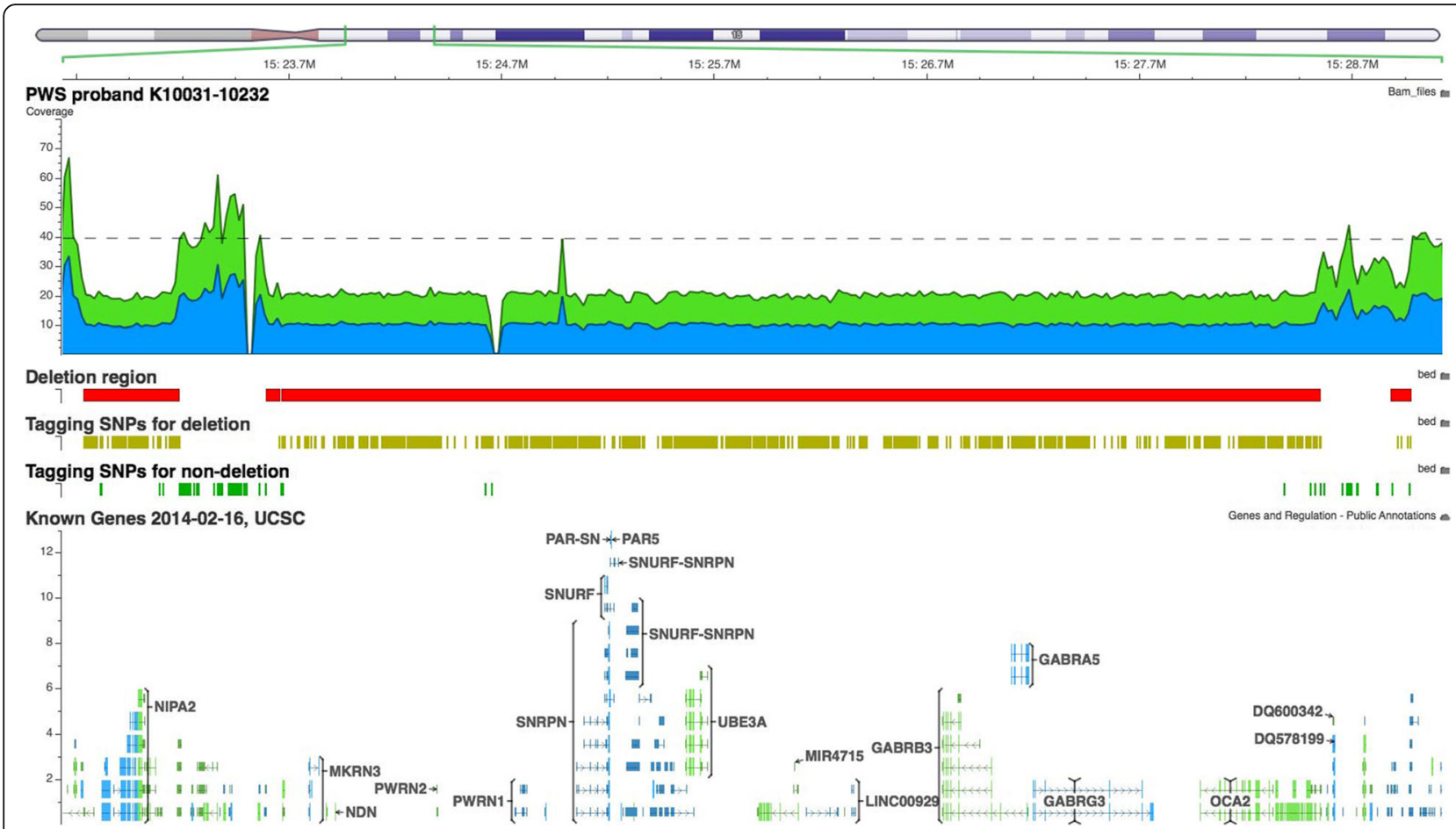

Fig. 4 Screenshot of three heterozygous de novo deletions between the region 15q11.2 to $15 q 13$ in proband K10031-10232. The deleted regions are denoted by the red boxes. The yellow tagging SNVs represent the SNVs that violate the Mendelian inheritance law. The non-deleted regions are denoted by the green tagging SNVs. Genome-wide average coverage (40X) is denoted by the grey dashed line. The breakpoints of these deletions (PWS Type I deletion) are chr15:22,749,401-23,198,800 ( 449 Kb), chr15:23,608,601-28,566,000 ( 4.96 Mb), and chr15:28,897,601-28,992,600 (95 Kb) (hg19). These deletions are not detected either in the proband's father or the unaffected brother

\section{Phenolyzer revealed the relationship between p.C282Y variant and $\mathrm{HH}$ in individual K10031-10145, which was missed by HPO analysis alone}

The mother (K10031-10145) with $\mathrm{HH}$ is homozygous for the p.C282Y variant in HFE, which is consistent with her molecular genetic assay results. Analyzing the HPO data with Phenomizer alone failed to suggest the diagnosis of $\mathrm{HH}$ (Additional file 1: Supplemental Data). This is likely because $\mathrm{HH}$ has many clinical features overlaping with other diseases. Even the most specific HPO term, HP:0011031 (abnormality of iron homeostasis), still links to 14 diseases and 9 genes. However, we were able to recover this finding when we used Phenolyzer to compare the patient's genomic and phenotypic profile. Phenolyzer pinpointed the only two out of eight input HPO terms which are indeed related to hemochromatosis, although these two terms are also reported with other diseases. As shown in Fig. 8, Phenolyzer successfully related the input HPO terms to hemochromatosis, as well as the input gene HFE. Because this was the most confident finding, the diagnosis of hemochromatosis was thus recommeneded by Phenolyzer.

Results from analyzing the WGS data showed that the mother's brother (K10031-10231) is also homozygous for the p.C282Y variant in HFE. However, his clinical test result has not yet provided any evidence to support the diagnosis of $\mathrm{HH}$, even though male p.C282Y homozygotes are considered more likely to develop ironoverload-related diseases due to the lack of the iron clearance events like menstruation and pregnancy in women [82]. This is in line with the fact that even family members can have variable expressivity of disease, including different onset ages, etc. This instance with the brother and the sister again highlights the point that the phenotypic expression of a given mutation in HFE may vary widely, influenced in part by unidentified modifier loci [83-89]. Some studies previously estimated that less than $1 \%$ of individuals in the U.S. carrying homozygous mutations present clearly with clinical diagnoses of hemochromatosis [90]. In contrast to studies that have searched for the "causal" gene, some have reported that genetic variations can instead have large effects on phenotypic variability, suggesting underlying genomic complexity from multiple interacting loci [91-94]. Understanding such diseases thus requires probabilistic thinking about the risk of developing the clinical manifestation, rather than deterministic genotype-phenotype "causation" [16, 95-97], and there will always be some 


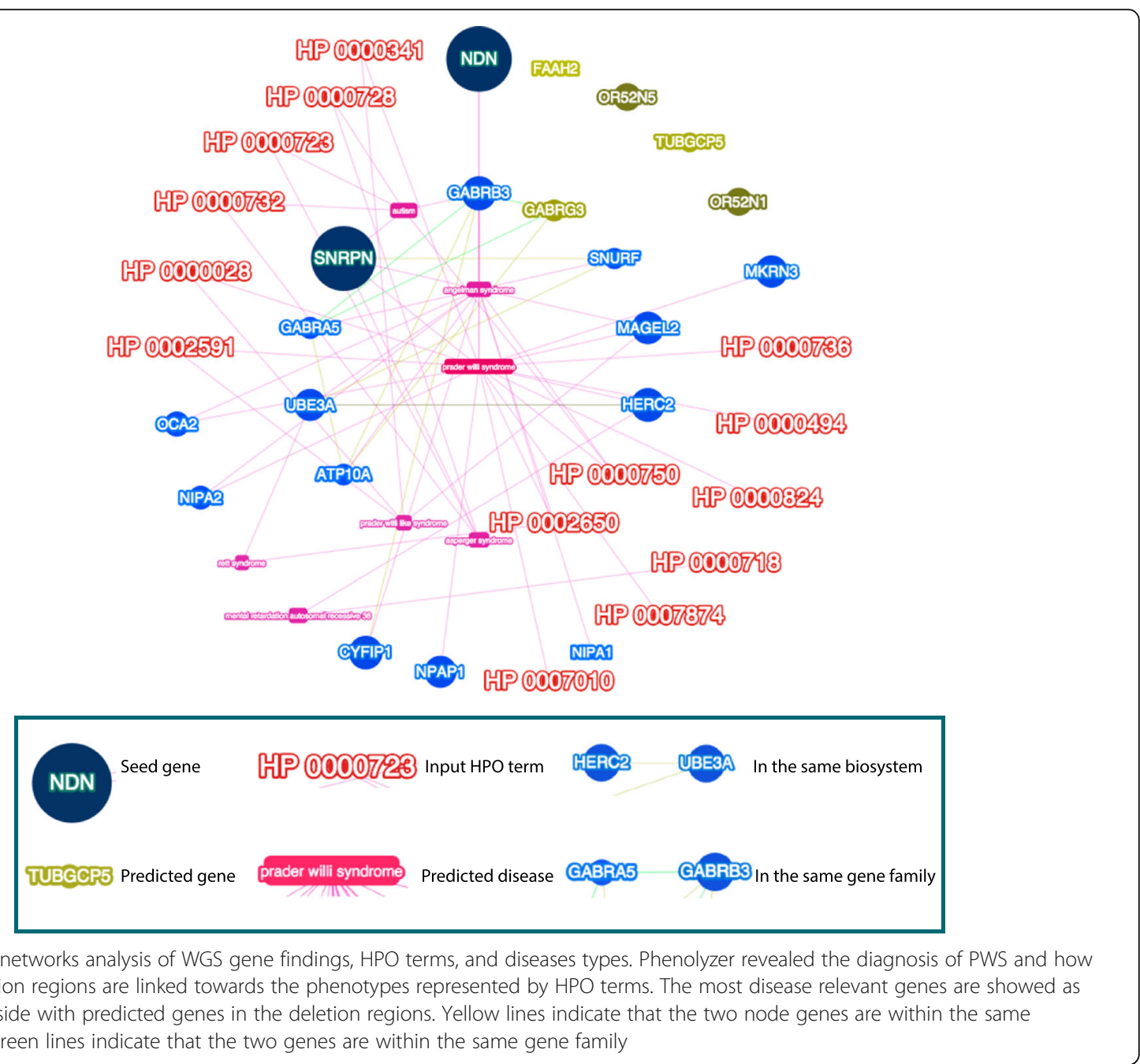

level of stochasticity as well [20]. Further, alongside the primary research-focused analysis, the participating subjects and families also received the research findings (Additional file 1: Table S8). Of course, we cannot exclude the possibility that we might have missed some variants, including possibly non-coding variants, and we expect that the future phenotyping, sequencing, and collation of data from millions of people will reveal associations that are not currently known.

\section{Analysis of dysautonomia-like symptoms}

None of the family members with dysautonomia-like symptoms carry any previously reported variants in $I K B K A P$ that are implicated in the autosomal recessive transmission of FD, which is also called hereditary sensory and autonomic neuropathy type III (HSAN-III). The WGS data have effective sequence coverage (> average coverage 40X) for this gene, but no novel rare variants were identified. Notably, both the mother (K1003110145) and the male proband (K10031-10138) carry heterozygous variants of p.H604Y and p.G613V in the protein product of NTRK1, which has been proven to contribute to HSAN-IV (congenital insensitivity to pain with anhidrosis). HSAN-IV is a disease closely resembling FD (HSAN III), and is characterized by a lack of pain sensation, anhidrosis, unexplained fever since childhood, and self-mutilating behavior $[98,99]$. Both variants are located within the intracellular tyrosine kinase domain of the encoded protein, but neither sites are conserved. Both variants have also been reported before in healthy individuals, so they are considered to be polymorphisms in the population and seem to be in linkage disequilibrium [100-104]. The mother's brother (K10031-10231, unaffected) also carries these two variants, so this provides further evidence that they are likely to be polymorphisms. Most importantly, neither variant is present in the proband K10031-10133, who reported the most severe dysautonomia-like symptoms.

Instead of the NTRK1 variant, a manual filtering found seven other putative variants in PLCG2, ATXN2, VWA8, 


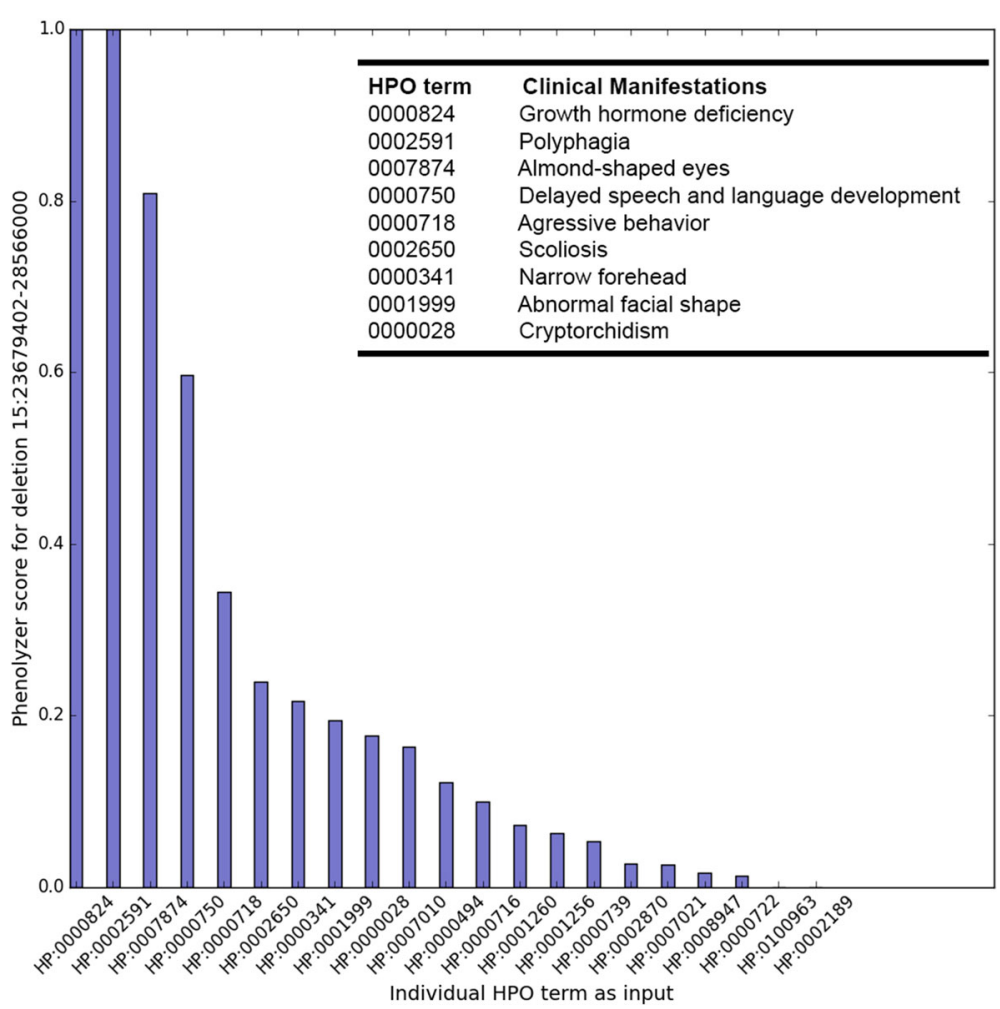

Fig. 6 Phenolyzer scores represent the importance of each HPO term for PWS diagnosis. Phenolyzer scores are normalized to a range from 0 to 1. A higher score indicates that this HPO term has a higher impact on the diagnosis of the corresponding disease. The clinical manifestations of the top HPO terms are shown in the figure legend

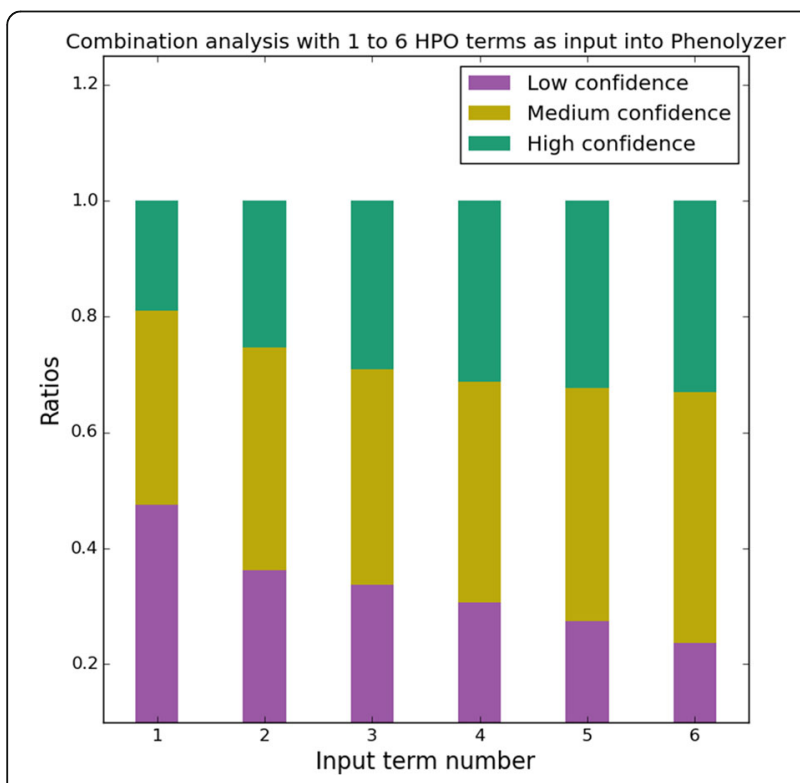

Fig. 7 Combination analysis indicates using more HPO terms lead to a higher chance of the correct prioritization. 'High confidence', where the known deletion has a normalized Phenolyzer score no less than 0.5; 'Medium confidence', where the known deletion has a normalized Phenolyzer score between 0.1 and 0.5 ; 'Low confidence', where the known deletion has a normalized Phenolyzer score less than 0.1
LRRIQ1, MYO1H, OR1J4, and RFX4 which follow a dominant inheritance model (Additional file 1: Table S4). Variants in PLCG2, ATXN2, and VWA8 were previously reported to be associated with certain disease phenotypes, including cold-induced urticaria, antibody deficiency,

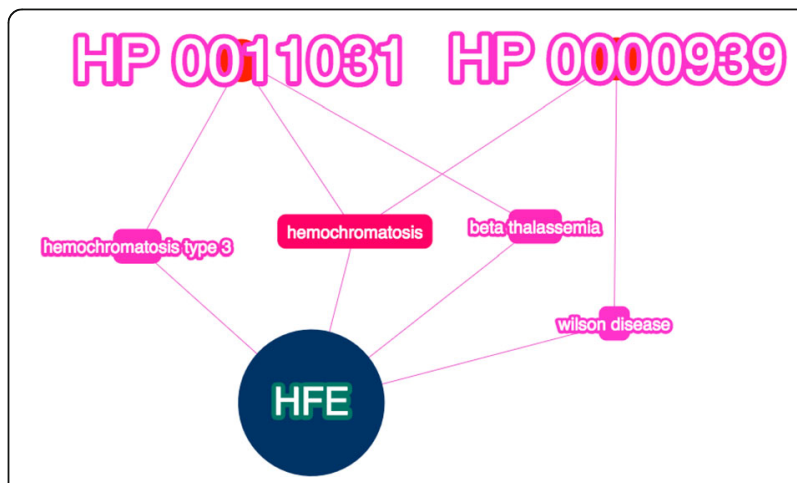

Fig. 8 Phenolyzer networks analysis of both HPO and WGS data yielded the correct diagnosis for the individual with HH (K1003110145). Phenolyzer successfully linked the gene HFE to two HPO terms (HP:0011031 Abnormality of iron homeostasis, HP:0000939 Osteoporosis), bridged by the predictive diagnosis, hemochromatosis. The most disease relevant gene HFE, is showed as a seed gene (blue) 
susceptibility to infection and autoimmunity, spinocerebellar ataxia type 2, celiac disease, and susceptibility to amyotrophic lateral sclerosis [98-100]. However, the variants we identified in this family are not the same variants in the literature, and all of these predicted diseases have only partially overlapping manifestations with dysautonomia-like symptoms. For the rest of the four genes mentioned above, LRRIQ1, MYO1H, OR1J4, and $R F X 4$, there has been, to our knowledge, no reports published to date discussing any variants in these genes associated with human disease. Therefore, the functional impact of these variants remains unclear.

Lastly, Phenolyzer analysis was carried out using the phenotype of proband K10031-10133 as input. It successfully prioritized two variants we identified in the manual filtering analysis discussed above, ATXN2 and $V W A 8$, and further revealed the complexity of such diseases (Additional file 1: Fig. S8).

These results together suggest that the genetic inheritance of dysautonomia-like symptoms in this pedigree may not consist of only one high-effect size mutation, but rather could be polygenic and/or environmentally influenced. It is possible that multiple variants including those we mentioned above are acting together or in conjunction with modifiers in these individuals' genomes to give rise to a spectrum of complex clinical manifestations.

\section{Conclusions}

This research report provides insights into using WGS as a genetic test to investigate PWS and other phenotypes. In our study, three de novo deletions were discovered at single base pair resolution. WGS enables the reconstruction of the recombination event in this imprinting hotspot 15q11-13, which provides deeper insights into the mechanism of PWS. Notably, this is the first report of an Illumina HiSeq WGS experiment on an individual with PWS with the paternal allele deletion. In principle, the use of WGS, once standardized, could eventually simplify the molecular diagnosis procedure for PWS and and other genetic syndrome patients, as one would no longer need to through the several steps for the standard genetic testing for PWS $[77,105,106]$. Since AS and PWS share a similar cytogenetic anomaly in $15 q 11.2$ to $15 q 13$ [107, 108], WGS could potentially help reveal the sub-types of both syndromes, given that the breakpoints of the CNVs can be mapped at the nucleotide level and one could distinguish which allele (paternal or maternal) has been deleted. However, WGS alone would not be enough to detect either uniparental paternal disomy with heterodisomy or imprinting defects in this genomic region for non-deleted PWS individuals [77, 109].

However, WGS might not always pinpoint the exact disease relevant variants, due to the limition of cohort size and disease complexity. Phenotype and genotype matching across cohorts is needed for confirming causal relationships. HPO has emerged as a standardized way to compare phenotypes, although it can only marginally solve the phenotype issue and cannot be directly used for WGS analysis. Fortunately, the development of phenotype-analysis tools such as Phenolyzer makes it possible to bridge the gap between the two on top of rich prior information across multiple databases. During the selection process for a particular patient's features, one is able to query a surplus of clinical and scientific knowledge about the diseases linked to the feature in question. In addition, integration of four types of gene-gene interaction databases in Phenolyzer makes it possible to find more candidate genes beyond the existing gene-disease knowledge and generate new biological hypotheses. While the common drawback of all the gene prediction tools is the balance between sensitivity and specificity, Phenolyzer uses a modified logistic regression model to address this problem, ensuring that well-established genes are recommended among a large set of predictions.

This report about integrating WGS and HPO data demonstrates the effectiveness of such an approach and shows its potential for clinical implementation. Neither technique on its own is ideal for clinical diagnosis, but fortunately they complement each other and thus help eliminate false positives and reveal novel insights into human diseases. The potential for HPO remains in the development of a more multi-dimensional depiction of subjects that takes into account the past and present human presentation, and will aid in efforts for early diagnoses and intervention. As the field of medical genetics advances, researchers will need to find an efficient way to capture phenotypic information that allows for the use of computational algorithms to search for phenotypic similarity between genomics studies [36]. For WGS, with ever-increasing sequencing capacity, a scalable and reliable informatic solution is key to analyzing millions of genomes simultaneously. To maximize this potential in clinical settings, data from WGS and HPO should be integrated and shared in a unified fashion.

\section{Additional files}

Additional file 1: Supplemental Tables and Figures. This file includes supplemental figures S1-S8, tables S1-S10, pharmacogenomic analyses for individual K10031-10133, additional clinical information of individuals in the study, and video descriptions. (PDF $1491 \mathrm{~kb}$ )

Additional file 2: Tute Genomics Report. This file contains nine Tute Genomics reports of all participants included in the study. (PDF $720 \mathrm{~kb}$ )

Additional file 3: Phenomizer Diagnosis Report. This file contains three Phenomizer diagnosis reports of individual K10031-10232, K10031-10133, and K10031-10145. (PDF 429 kb) 


\section{Abbreviations}

bp: Base pair; CIPA: Congenital insensitivity to pain with anhidrosis; CNV: Copy number variation; FD: Familial dysautonomia; HH: Hereditary hemochromatosis; HPO: The human phenotype ontology; INDELs: Insertions and deletions; Kb: Kilo base pairs; Mb: Megabase pairs; NGS: Next-generation sequencing; OCD: Obsessive-compulsive disorder; PCR: Polymerase chain reaction; PWS: Prader-Willi syndrome; SNV: Single-nucleotide variation; SV: Structual variant; TS: Tourette syndrome; WES: Whole exome sequencing; WGS: Whole genome sequencing

\section{Acknowledgements}

The authors acknowledge Gareth Highnam and Jason O'Rawe for bioinformatics support and comments on the manuscript. The authors would like to thank the Exome Aggregation Consortium and the groups that provided exome variant data for comparison. A full list of contributing groups can be found at http://exac.broadinstitute.org/about.

\section{Funding}

The laboratory of G.J.L. is supported by funds from the Stanley Institute for Cognitive Genomics at Cold Spring Harbor Laboratory (CSHL). The CSHL genome center is supported in part by a Cancer Center Support Grant (CA045508) from the NCI. K.W. is supported by NIH grant HG006465.

\section{Availability of data and materials}

All of the sequence reads can be downloaded under project accession number [SRP058003] from the Sequence Read Archive (http:// www.ncbi.nlm.nih.gov/sra). Administrative permission was received from the Utah Foundation for Biomedical Research to access the medical records reviewed in this study.

\section{Authors' contributions}

GJL and RR helped to recruit the family and conduct clinical phenotyping. HF analyzed the sequencing data. YW analyzed the clinical data, performed Sanger sequencing validation experiment, assisted in the WGS experiment and the HPO analysis. HY conducted the computational analysis with Phenolyzer. MY performed the HPO analysis and helped with the clinical data analysis. $\perp B$ and DM helped analyze the WGS and microarray data. HF, YW, HY, MY and GJL wrote the manuscript. KW and GJL supervised the data analysis. All of the authors have read and approved the final manuscript.

\section{Competing interests}

G.J.L serves on advisory boards for GenePeeks, Inc., Omicia, Inc., and Seven Bridges Genomics, Inc., is a consultant to Genos, Inc., and previously served as a consultant to Good Start Genetics, Inc. R.R. and K.W. were board members and shareholders of Tute Genomics, Inc. D.M. was an investor in Tute Genomics.

\section{Consent for publication}

Written consent was received from all study subjects (parental consent in children under the age of 18) to publish their personal and clinical details relevant to the study including parents' ethnicity, along video and facial photography provided upon request to qualified investigators.

\section{Ethics approval and consent to participate}

The collection and analysis of the DNA used in this study was conducted by the Utah Foundation for Biomedical Research, Protocol \#100, approved by Ethical \& Independent Review Services, Inc. Written informed consent (parental consent in children under the age of 18) to participate in research including sample collection was obtained from all participants in the study. Research was carried out in compliance with the Federal Policy for the Protection of Human Subjects 45C.F.R.46.

\section{Online resources}

The Human Phenotype Ontology (HPO): http://human-phenotypeontology.github.io/page2/ 1000 G database: http://www.1000genomes.org/ Exome Aggregation Consortium (ExAC): http://exac.broadinstitute.org/ ClinVar database: http://www.ncbi.nlm.nih.gov/clinvar/ Phenolyzer: phenolyzer.wglab.org

\section{Author details}

'Stanley Institute for Cognitive Genomics, One Bungtown Road, Cold Spring Harbor Laboratory, Cold Spring Harbor, NY, USA. ${ }^{2}$ Stony Brook University, 100 Nicolls Rd, Stony Brook, NY, USA. ${ }^{3}$ Simons Center for Quantitative Biology, One Bungtown Road, Cold Spring Harbor Laboratory, Cold Spring Harbor, NY, USA. ${ }^{4}$ Centro de Ciencias Genomicas, Universidad Nacional Autonoma de Mexico, Cuernavaca, Morelos, MX, Mexico. ${ }^{5}$ Tute, Genomics Inc., 150 S 100 W, Provo, UT, USA. ${ }^{6}$ Utah Foundation for Biomedical Research, Salt Lake City, UT, USA. ${ }^{7}$ Zilkha Neurogenetic Institute, University of Southern California, Los Angeles, CA, USA. ${ }^{8}$ Neuroscience Graduate Program, University of Southern California, Los Angeles, CA, USA. ${ }^{9}$ Department of Psychiatry, University of Southern California, Los Angeles, CA, USA. ${ }^{10}$ Division of Bioinformatics, Department of Preventive Medicine, University of Southern California, Los Angeles, CA, USA. ${ }^{11}$ Present Address: Department of Biomedical Informatics and Institute for Genomic Medicine, Columbia University Medical Center, New York 10032, NY, USA.

Received: 28 September 2016 Accepted: 14 February 2017

Published online: 23 February 2017

\section{References}

1. Meijers-Heijboer EJ, Verhoog LC, Brekelmans CTM, Seynaeve C, TilanusLinthorst MMA, Wagner A, Dukel L, Devilee P, van den Ouweland AMW, van Geel AN, et al. Presymptomatic DNA testing and prophylactic surgery in families with a BRCA1 or BRCA2 mutation. Lancet. 2000;355(9220):2015-20.

2. Nanda R, Schumm L, Cummings $S$, et al. Genetic testing in an ethnically diverse cohort of high-risk women: a comparative analysis of brca1 and brca2 mutations in american families of european and African ancestry. JAMA. 2005;294(15):1925-33.

3. Sherman S, Pletcher BA, Driscoll DA. Fragile $X$ syndrome: diagnostic and carrier testing. Genet Med. 2005;7(8):584-7.

4. Walker FO. Huntington's disease. Lancet. 2007;369(9557):218-28.

5. Palomaki GE, Kloza EM, Lambert-Messerlian GM, Haddow JE, Neveux LM, Ehrich M, van den Boom D, Bombard AT, Deciu C, Grody WW, et al. DNA sequencing of maternal plasma to detect down syndrome: an international clinical validation study. Genet Med. 2011;13(11):913-20.

6. Morton CC, Nance WE. Newborn hearing screening - a silent revolution. N Engl J Med. 2006;354(20):2151-64.

7. Thompson DC, McPhillips H, Davis RL, Lieu TA, Homer CJ, Helfand M. Universal newborn hearing screening: summary of evidence. JAMA. 2001;286(16):2000-10.

8. Evans JP, Skrzynia C, Burke W. The complexities of predictive genetic testing. BMJ Br Med J. 2001;322(7293):1052-6.

9. Smith GD, Ebrahim S, Lewis S, Hansell AL, Palmer LJ, Burton PR. Genetic epidemiology and public health: hope, hype, and future prospects. Lancet. 2005:366(9495):1484-98.

10. Rope Alan F, Wang K, Evjenth R, Xing J, Johnston Jennifer J, Swensen Jeffrey J, Johnson WE, Moore B, Huff Chad D, Bird Lynne M, et al. Using VAAST to identify an X-linked disorder resulting in lethality in male infants Due to N-terminal acetyltransferase deficiency. Am J Hum Genet. 2011;89(1):28-43.

11. Honeyman JN, Simon EP, Robine N, Chiaroni-Clarke R, Darcy DG, Lim IIP, Gleason CE, Murphy JM, Rosenberg BR, Teegan L, et al. Detection of a recurrent DNAJB1-PRKACA chimeric transcript in fibrolamellar hepatocellular carcinoma. Science. 2014:343(6174):1010-4.

12. Boycott KM, Vanstone MR, Bulman DE, MacKenzie AE. Rare-disease genetics in the era of next-generation sequencing: discovery to translation. Nat Rev Genet. 2013;14(10):681-91.

13. Koboldt Daniel C, Steinberg Karyn M, Larson David E, Wilson Richard K, Mardis ER. The next-generation sequencing revolution and its impact on genomics. Cell. 2013;155(1):27-38.

14. Bamshad MJ, Ng SB, Bigham AW, Tabor HK, Emond MJ, Nickerson DA Shendure J. Exome sequencing as a tool for Mendelian disease gene discovery. Nat Rev Genet. 2011;12(11):745-55.

15. Lee $H$, Deignan JL, Dorrani $N$, et al. CLinical exome sequencing for genetic identification of rare mendelian disorders. JAMA. 2014;312(18):1880-7.

16. Lyon GJ, O'Rawe J. Human genetics and clinical aspects of neurodevelopmental disorders. In: Mitchell K, editor. The Genetics of Neurodevelopmental Disorders. Hoboken: Wiley-Blackwell; 2015. p. 368. ISBN: 978-1-118-52488-6. 
17. Grillo E, Lo Rizzo C, Bianciardi L, Bizzarri V, Baldassarri M, Spiga O, Furini S, De Felice C, Signorini C, Leoncini $S$, et al. Revealing the complexity of a monogenic disease: rett syndrome exome sequencing. PLoS One. 2013:8(2):e56599.

18. Hamilton BA, Yu BD. Modifier genes and the plasticity of genetic networks in mice. PLoS Genet. 2012;8(4):e1002644.

19. Li H, Cherry S, Klinedinst D, DeLeon V, Redig J, Reshey B, Chin MT, Sherman SL, Maslen $\mathrm{CL}$, Reeves $\mathrm{RH}$. Genetic modifiers predisposing to congenital heart disease in the sensitized down syndrome population. Circ Cardiovasc Genet. 2012;5(3):301-8.

20. Vogt G. Stochastic developmental variation, an epigenetic source of phenotypic diversity with far-reaching biological consequences. J Biosci. 2015:40(1):159-204.

21. Lyon GJ, Segal JP. Practical, ethical and regulatory considerations for the evolving medical and research genomics landscape. Appl Transl Genom. 2013;2:34-40.

22. O'Rawe J, Jiang T, Sun G, Wu Y, Wang W, Hu J, Bodily P, Tian L, Hakonarson $H$, Johnson WE, et al. Low concordance of multiple variant-calling pipelines: practical implications for exome and genome sequencing. Genome Med. 2013;5:1-18

23. Lyon GJ. Personalized medicine: bring clinical standards to human-genetics research. Nature. 2012;482(7385):300-1.

24. Dewey FE, Grove ME, Pan C, et al. CLinical interpretation and implications of whole-genome sequencing. JAMA. 2014;311(10):1035-45.

25. Slaugenhaupt SA, Blumenfeld A, Gill SP, Leyne M, Mull J, Cuajungco MP Liebert CB, Chadwick B, Idelson M, Reznik L, et al. Tissue-specific expression of a splicing mutation in the IKBKAP gene causes familial Dysautonomia. Am J Hum Genet. 2001;68(3):598-605.

26. Esteller M. Non-coding RNAs in human disease. Nat Rev Genet. 2011:12(12):861-74

27. Pagani F, Stuani C, Tzetis M, Kanavakis E, Efthymiadou A, Doudounakis S, Casals T, Baralle FE. New type of disease causing mutations: the example of the composite exonic regulatory elements of splicing in CFTR exon 12. Hum Mol Genet. 2003;12(10):1111-20.

28. Wang G-S, Cooper TA. Splicing in disease: disruption of the splicing code and the decoding machinery. Nat Rev Genet. 2007;8(10):749-61.

29. Faustino NA, Cooper TA. Pre-mRNA splicing and human disease. Genes Dev. 2003;17(4):419-37.

30. Venables JP. Aberrant and alternative splicing in cancer. Cancer Res. 2004;64(21):7647-54.

31. Weischenfeldt J, Symmons O, Spitz F, Korbel JO. Phenotypic impact of genomic structural variation: insights from and for human disease. Nat Rev Genet. 2013;14(2):125-38.

32. Day-Williams AG, Sun C, Jelcic I, McLaughlin H, Harris T, Martin R, Carulli J. Whole genome sequencing reveals a chromosome $9 p$ deletion causing DOCK8 deficiency in an adult diagnosed with hyper lgE syndrome Who developed progressive multifocal leukoencephalopathy. J Clin Immunol. 2015;35(1):92-6.

33. Wang K, Kim C, Bradfield J, Guo Y, Toskala E, Otieno F, Hou C, Thomas K, Cardinale $\mathrm{C}$, Lyon $\mathrm{G}$, et al. Whole-genome DNARNA sequencing identifies truncating mutations in RBCK1 in a novel Mendelian disease with neuromuscular and cardiac involvement. Genome Med. 2013;5(7):67.

34. Fang H, Wu Y, Narzisi G, O'Rawe J, Barrón LTJ, Rosenbaum J, Ronemus M, lossifov I, Schatz M, Lyon G. Reducing INDEL calling errors in whole-genome and exome sequencing data. Genome Med. 2014;6:89.

35. Robinson PN, Köhler S, Bauer S, Seelow D, Horn D, Mundlos S. The human phenotype ontology: a tool for annotating and analyzing human hereditary disease. Am J Hum Genet. 2008;83(5):610-5.

36. Robinson PN, Mundlos S. The human phenotype ontology. Clin Genet. 2010;77(6):525-34

37. Kohler S, Vasilevsky NA, Engelstad M, Foster E, McMurry J, Ayme S, Baynam G, Bello SM, Boerkoel CF, Boycott KM, et al. The human phenotype ontology in 2017. Nucleic Acids Res. 2017;45(D1):D865-76

38. Köhler S, Schoeneberg U, Czeschik JC, Doelken SC, Hehir-Kwa JY, Ibn-Salem J, Mungall CJ, Smedley D, Haendel MA, Robinson PN: Clinical interpretation of CNVs with cross-species phenotype data. J Med Genet 2014;51(11):766-72. doi:10.1136/jmedgenet-2014-102633. Epub 2014 Oct 3.

39. Robinson PN, Köhler S, Oellrich A, Project SMG, Wang K, Mungall CJ, Lewis SE, Washington N, Bauer S, Seelow D, et al. Improved exome prioritization of disease genes through cross-species phenotype comparison. Genome Res. 2014;24(2):340-8.
40. Smedley D, Robinson PN. Phenotype-driven strategies for exome prioritization of human Mendelian disease genes. Genome Med. 2015;7(1):81.

41. Zemojtel $T$, Kohler S, Mackenroth $L$, Jager $M$, Hecht J, Krawitz P, Graul-Neumann L, Doelken S, Ehmke N, Spielmann M, et al. Effective diagnosis of genetic disease by computational phenotype analysis of the disease-associated genome. Sci Transl Med. 2014;6(252):252ra123.

42. Smedley $D$, Schubach $M$, Jacobsen JO, Kohler S, Zemojtel T, Spielmann $M$, Jager $M$, Hochheiser $H$, Washington NL, McMurry JA, et al. A wholegenome analysis framework for effective identification of pathogenic regulatory variants in Mendelian disease. Am J Hum Genet. 2016;99(3):595-606.

43. Yang $H$, Robinson PN, Wang K. Phenolyzer: phenotype-based prioritization of candidate genes for human diseases. Nat Methods. 2015;12(9):841-3.

44. Hurren BJ, Flack NA. Prader-Willi syndrome: a spectrum of anatomical and clinical features. Clin Anat. 2016;29(5):590-605.

45. Butler MG, Manzardo AM, Forster JL. Prader-Willi syndrome: clinical genetics and diagnostic aspects with treatment approaches. Curr Pediatr Rev. 2016;12(2):136-66.

46. Georgitsi M, Willsey AJ, Mathews CA, State M, Scharf JM, Paschou P. The genetic etiology of Tourette syndrome: large-scale collaborative efforts on the precipice of discovery. Front Neurosci. 2016;10:351.

47. Slinkard JM. Multiple sleep latency test and maintenance of wakefulness test. Respir Care Clin N Am. 2006;12(1):17-22. viii.

48. Freeman BJ, Ritvo ER, Schroth PC. Behavior assessment of the syndrome of autism: behavior observation system. J Am Acad Child Psychiatry. 1984;23(5):588-94.

49. Moulton E, Bradbury K, Barton M, Fein D: Factor Analysis of the Childhood Autism Rating Scale in a Sample of Two Year Olds with an Autism Spectrum Disorder. J Autism Dev Disord. 2016. [Epub ahead of print]

50. Song J, Leventhal BL, Koh YJ, Cheon KA, Hong HJ, Kim YK, Cho K, Lim EC, Park JI, Kim YS. Cross-cultural aspect of behavior assessment system for children-2, parent rating scale-child: standardization in Korean children. Yonsei Med J. 2017:58(2):439-48.

51. Dean CE, Russell JM, Kuskowski MA, Caligiuri MP, Nugent SM. Clinical rating scales and instruments: how do they compare in assessing abnormal, involuntary movements? J Clin Psychopharmacol. 2004;24(3):298-304.

52. Li H. Aligning sequence reads, clone sequences and assembly contigs with BWA-MEM, ArXiv e-prints, vol. 1303. 2013. p. 3997.

53. Li H, Handsaker B, Wysoker A, Fennell T, Ruan J, Homer N, Marth G, Abecasis G, Durbin R, Subgroup GPDP. The sequence alignment/Map format and SAMtools. Bioinformatics. 2009;25(16):2078-9.

54. McKenna A, Hanna M, Banks E, Sivachenko A, Cibulskis K, Kernytsky A, Garimella K, Altshuler D, Gabriel S, Daly M, et al. The genome analysis toolkit: a MapReduce framework for analyzing next-generation DNA sequencing data. Genome Res. 2010;20(9):1297-303.

55. García-Alcalde F, Okonechnikov K, Carbonell J, Cruz LM, Götz S, Tarazona S, Dopazo J, Meyer TF, Conesa A. Qualimap: evaluating next-generation sequencing alignment data. Bioinformatics. 2012;28(20):2678-9.

56. O'Rawe J, Jiang T, Sun G, Wu Y, Wang W, Hu J, Bodily P, Tian L, Hakonarson H, Johnson WE, et al. Low concordance of multiple variant-calling pipelines: practical implications for exome and genome sequencing. Genome Med. 2013;5(3):28.

57. Jimenez-Barron LT, O'Rawe JA, Wu Y, Yoon M, Fang H, lossifov I, Lyon GJ. Genome-wide variant analysis of simplex autism families with an integrative clinical-bioinformatics pipeline. Cold Spring Harb Mol Case Stud. 2015;1(1):a000422.

58. DePristo MA, Banks E, Poplin R, Garimella KV, Maguire JR, Hartl C, Philippakis AA, del Angel G, Rivas MA, Hanna M, et al. A framework for variation discovery and genotyping using next-generation DNA sequencing data. Nat Genet. 2011;43(5):491-8.

59. Garrison E, Marth G. Haplotype-based variant detection from short-read sequencing, ArXiv e-prints, vol. 1207. 2012. p. 3907.

60. Narzisi G, O'Rawe JA, lossifov I, Fang H, Lee Y-h, Wang Z, Wu Y, Lyon GJ, Wigler M, Schatz MC: Accurate de novo and transmitted indel detection in exome-capture data using microassembly. Nat Meth. Advance online publication. 2014;11(10):1033-6.

61. Highnam G, Franck C, Martin A, Stephens C, Puthige A, Mittelman D. Accurate human microsatellite genotypes from high-throughput resequencing data using informed error profiles. Nucleic Acids Res. 2013;41(1):e32. 
62. Layer R, Chiang C, Quinlan A, Hall I. LUMPY: a probabilistic framework for structural variant discovery. Genome Biol. 2014;15(6):R84.

63. Abyzov A, Urban AE, Snyder M, Gerstein M. CNVnator: an approach to discover, genotype, and characterize typical and atypical CNVs from family and population genome sequencing. Genome Res. 2011;21(6):974-84

64. Zhu M, Need AC, Han Y, Ge D, Maia JM, Zhu Q, Heinzen EL, Cirulli ET, Pelak K, He $M$, et al. Using ERDS to infer copy-number variants in high-coverage genomes. Am J Hum Genet. 2012;91(3):408-21.

65. Wang K, Li M, Hadley D, Liu R, Glessner J, Grant SFA, Hakonarson H, Bucan M. PennCNV: An integrated hidden Markov model designed for high-resolution copy number variation detection in whole-genome SNP genotyping data. Genome Res. 2007;17(11):1665-74.

66. Quinlan AR, Hall IM. BEDTools: a flexible suite of utilities for comparing genomic features. Bioinformatics. 2010;26(6):841-2.

67. Paila U, Chapman BA, Kirchner R, Quinlan AR. GEMINI: integrative exploration of genetic variation and genome annotations. PLoS Comput Biol. 2013;9(7):e1003153.

68. Wang K, Li M, Hakonarson H. ANNOVAR: functional annotation of genetic variants from high-throughput sequencing data. Nucleic Acids Res. 2010;38(16):e164.

69. Gu Z, Gu L, Eils R, Schlesner M, Brors B. circlize implements and enhances circular visualization in R. Bioinformatics. 2014:30(19):2811-2.

70. Lek M, Karczewski KJ, Minikel EV, Samocha KE, Banks E, Fennell T, O'Donnell-Luria AH, Ware JS, Hill AJ, Cummings BB, et al. Analysis of protein-coding genetic variation in 60,706 humans. Nature. 2016; 536(7616):285-91.

71. MacArthur DG, Balasubramanian S, Frankish A, Huang N, Morris J, Walter K, Jostins L, Habegger L, Pickrell JK, Montgomery SB, et al. A systematic survey of loss-of-function variants in human protein-coding genes. Science. 2012;335(6070):823-8.

72. Kircher M, Witten DM, Jain P, O'Roak BJ, Cooper GM, Shendure J. A general framework for estimating the relative pathogenicity of human genetic variants. Nat Genet. 2014;46(3):310-5

73. Hu H, Roach JC, Coon H, Guthery SL, Voelkerding KV, Margraf RL, Durtschi JD, Tavtigian SV, Shankaracharya, Wu W, et al. A unified test of linkage analysis and rare-variant association for analysis of pedigree sequence data. Nat Biotechnol. 2014;32(7):663-9.

74. Kohler S, Schulz MH, Krawitz P, Bauer S, Dolken S, Ott CE, Mundlos C, Horn D, Mundlos S, Robinson PN. Clinical diagnostics in human genetics with semantic similarity searches in ontologies. Am J Hum Genet. 2009;85(4):457-64.

75. Kohler S, Doelken SC, Mungall CJ, Bauer S, Firth HV, Bailleul-Forestier I, Black GC, Brown DL, Brudno M, Campbell J, et al. The human phenotype ontology project: linking molecular biology and disease through phenotype data. Nucleic Acids Res. 2014;42(Database issue):D966-74.

76. Manichaikul A, Mychaleckyj JC, Rich SS, Daly K, Sale M, Chen W-M. Robust relationship inference in genome-wide association studies. Bioinformatics. 2010;26(22):2867-73

77. Cassidy SB, Schwartz S, Miller JL, Driscoll DJ. Prader-Willi syndrome. Genet Med. 2012;14(1):10-26.

78. Christian SL, Robinson WP, Huang B, Mutirangura A, Line MR, Nakao M, Surti U, Chakravarti A, Ledbetter DH. Molecular characterization of two proximal deletion breakpoint regions in both Prader-Willi and angelman syndrome patients. Am J Hum Genet. 1995;57(1):40-8.

79. Reed ML, Leff SE. Maternal imprinting of human SNRPN, a gene deleted in Prader-Willi syndrome. Nat Genet. 1994;6(2):163-7.

80. Jay $P$, Rougeulle C, Massacrier A, Moncla A, Mattel M-G, Malzac P, Roeckel N, Taviaux S, Berge Lefranc J-L, Cau P, et al. The human necdin gene, NDN, is maternally imprinted and located in the Prader-Willi syndrome chromosomal region. Nat Genet. 1997;17(3):357-61.

81. Fang P, Lev-Lehman E, Tsai TF, Matsuura T, Benton CS, Sutcliffe JS, Christian SL, Kubota T, Halley DJ, Meijers-Heijboer $H$, et al. The spectrum of mutations in UBE3A causing angelman syndrome. Hum Mol Genet. 1999;8(1):129-35.

82. Allen KJ, Gurrin LC, Constantine CC, Osborne NJ, Delatycki MB, Nicoll AJ, McLaren CE, Bahlo M, Nisselle AE, Vulpe CD, et al. Iron-overloadrelated disease in HFE hereditary hemochromatosis. N Engl J Med. 2008;358(3):221-30.

83. Pietrangelo A. Hereditary hemochromatosis - a New look at an Old disease. N Engl J Med. 2004;350(23):2383-97.
84. Hanson EH, Imperatore G, Burke W. HFE gene and hereditary hemochromatosis: a HuGE review. Am J Epidemiol. 2001;154(3):193-206.

85. McLaren CE, Emond MJ, Subramaniam VN, Phatak PD, Barton JC, Adams PC, Powell LW, Gurrin LC, Ramm GA, Anderson GJ et al.: Exome sequencing in HFE C282Y homozygous men with extreme phenotypes identifies a GNPAT variant associated with severe iron overload. Hepatology. 2015;62(2):429-39.

86. Pelucchi S, Mariani R, Calza S, Fracanzani AL, Modignani GL, Bertola F, Busti F, Trombini P, Fraquelli M, Forni GL, et al. CYBRD1 as a modifier gene that modulates iron phenotype in HFE p.C282Y homozygous patients. Haematologica. 2012;97(12):1818-25.

87. Stickel F, Buch S, Zoller H, Hultcrantz R, Gallati S, Osterreicher C, Finkenstedt A, Stadlmayr A, Aigner E, Sahinbegovic E, et al. Evaluation of genome-wide loci of iron metabolism in hereditary hemochromatosis identifies PCSK7 as a host risk factor of liver cirrhosis. Hum Mol Genet. 2014;23(14):3883-90.

88. Triess C, von Figura G, Stuhrmann M, Butzeck B, Krayenbuehl PA, Strnad P, Kulaksiz H. Diagnosis of hereditary hemochromatosis in the era of genetic testing. Dig Dis Sci. 2012;57(11):2988-94.

89. Valenti L, Fracanzani AL, Rametta R, Fraquelli M, Soverini G, Pelusi S, Dongiovanni P, Conte D, Fargion S. Effect of the A736V TMPRSS6 polymorphism on the penetrance and clinical expression of hereditary hemochromatosis. J Hepatol. 2012;57(6):1319-25.

90. Beutler E, Felitti VJ, Koziol JA, Ho NJ, Gelbart T. Penetrance of $845 \mathrm{G} \rightarrow \mathrm{A}$ (C282Y) HFE hereditary haemochromatosis mutation in the USA. Lancet. 2002;359(9302):211-8.

91. Massouras A, Waszak SM, Albarca-Aguilera M, Hens K, Holcombe W, Ayroles JF, Dermitzakis ET, Stone EA, Jensen JD, Mackay TFC, et al. Genomic variation and its impact on gene expression in $<$ italic $>$ drosophila melanogaster</italic> PLoS Genet. 2012;8(11):e1003055.

92. Corbett-Detig RB, Zhou J, Clark AG, Hartl DL, Ayroles JF. Genetic incompatibilities are widespread within species. Nature. 2013;504(7478):135-7.

93. Mackay TFC, Stone EA, Ayroles JF. The genetics of quantitative traits: challenges and prospects. Nat Rev Genet. 2009;10(8):565-77.

94. Zuk O, Hechter E, Sunyaev SR, Lander ES. The mystery of missing heritability: genetic interactions create phantom heritability. Proc Natl Acad Sci U S A. 2012;109(4):1193-8.

95. Moczulski DK, Grzeszczak W, Gawlik B. Role of hemochromatosis C282Y and H63D mutations in HFE gene in development of type 2 diabetes and diabetic nephropathy. Diabetes Care. 2001;24(7):1187-91.

96. Thornton-Wells TA, Moore JH, Haines JL. Genetics, statistics and human disease: analytical retooling for complexity. Trends Genet. 2004;20(12):640-7.

97. Freund J, Brandmaier AM, Lewejohann L, Kirste I, Kritzler M, Krüger A, Sachser N, Lindenberger U, Kempermann G. Emergence of individuality in genetically identical mice. Science. 2013;340(6133):756-9.

98. Swanson AG. Congenital insensitivity to pain with anhydrosis: a unique syndrome in two male siblings. Arch Neurol. 1963;8(3):299-306.

99. Indo Y, Tsuruta M, Hayashida Y, Karim MA, Ohta K, Kawano T, Mitsubuchi H, Tonoki H, Awaya Y, Matsuda I. Mutations in the TRKA/NGF receptor gene in patients with congenital insensitivity to pain with anhidrosis. Nat Genet. 1996;13(4):485-8.

100. Gimm O, Greco A, Hoang-Vu C, Dralle H, Pierotti MA, Eng C. Mutation analysis reveals novel sequence variants in NTRK1 in sporadic human medullary thyroid carcinoma. J Clin Endocrinol Metab. 1999;84(8):2784-7.

101. Cargill M, Altshuler D, Ireland J, Sklar P, Ardlie K, Patil N, Lane CR, Lim EP, Kalyanaraman N, Nemesh J, et al. Characterization of single-nucleotide polymorphisms in coding regions of human genes. Nat Genet. 1999;22(3):231-8

102. Shatzky S, Moses S, Levy J, Pinsk V, Hershkovitz E, Herzog L, Shorer Z, Luder A, Parvari R. Congenital insensitivity to pain with anhidrosis (CIPA) in Israeli-Bedouins: genetic heterogeneity, novel mutations in the TRKA/ NGF receptor gene, clinical findings, and results of nerve conduction studies. Am J Med Genet. 2000;92(5):353-60.

103. Greenman C, Stephens P, Smith R, Dalgliesh GL, Hunter C, Bignell G, Davies $H$, Teague J, Butler A, Stevens C, et al. Patterns of somatic mutation in human cancer genomes. Nature. 2007;446(7132):153-8.

104. Indo Y. Molecular basis of congenital insensitivity to pain with anhidrosis (CIPA): mutations and polymorphisms in TRKA (NTRK1) gene encoding the receptor tyrosine kinase for nerve growth factor. Hum Mutat. 2001;18(6):462-71

105. Dittrich B, Robinson W, Knoblauch H, Buiting K, Schmidt K, GillessenKaesbach G, Horsthemke B. Molecular diagnosis of the Prader-Willi and 
angelman syndromes by detection of parent-of-origin specific DNA methylation in 15q11-13. Hum Genet. 1992;90(3):313-5.

106. Cassidy SB, Driscoll DJ. Prader-Willi syndrome. Eur J Hum Genet. 2008;17(1):3-13.

107. Knoll JHM, Nicholls RD, Magenis RE, Graham JM, Lalande M, Latt SA, Opitz JM, Reynolds JF. Angelman and Prader-Willi syndromes share a common chromosome 15 deletion but differ in parental origin of the deletion. Am J Med Genet. 1989;32(2):285-90.

108. Nicholls RD, Knepper JL. Genome organization, function, and imprinting in Prader-Willi and angelman syndromes. Annu Rev Genomics Hum Genet. 2001;2(1):153-75.

109. Malcolm S, Clayton-Smith J, Nichols M, Pembrey ME, Armour JAL, Jeffreys AJ, Robb S, Webb T. Uniparental paternal disomy in Angelman's syndrome. Lancet. 1991;337(8743):694-7.

Submit your next manuscript to BioMed Central and we will help you at every step:

- We accept pre-submission inquiries

- Our selector tool helps you to find the most relevant journal

- We provide round the clock customer support

- Convenient online submission

- Thorough peer review

- Inclusion in PubMed and all major indexing services

- Maximum visibility for your research

Submit your manuscript at www.biomedcentral.com/submit
Biomed Central 